\title{
Septic Arthritis of the Hand during Treatment of Complex Regional Pain Syndrome
}

\author{
Kentaro Tsuji, Hiroshi Takahashi, Ryoichi Fukano, Masayuki Sekiguchi \\ Department of Orthopedic Surgery, Toho University School of Medicine, Tokyo, Japan \\ Email: kt_hero0414@yahoo.co.jp
}

How to cite this paper: Tsuji, K., Takahashi, H., Fukano, R. and Sekiguchi, M. (2018) Septic Arthritis of the Hand during Treatment of Complex Regional Pain Syndrome. Open Journal of Orthopedics, $\mathbf{8}$, 317-321.

https://doi.org/10.4236/ojo.2018.88034

Received: July 28, 2018

Accepted: August 14, 2018

Published: August 17, 2018

Copyright ( 2018 by authors and Scientific Research Publishing Inc. This work is licensed under the Creative Commons Attribution International License (CC BY 4.0).

http://creativecommons.org/licenses/by/4.0/

\begin{abstract}
Complex regional pain syndrome (CRPS) complicated with septic arthritis of the hand has not previously been described. We treated a patient in whom swelling of the wrist recurred during treatment of CRPS and septic arthritis of the hand was induced by group $\mathrm{G}$ hemolytic streptococcus. There are case reports of tuberculous arthritis of the hand during treatment of CRPS; however, the causal relationship of wrist joint inflammation with CRPS is unclear. Careful follow-up is necessary for wrist joint inflammation if a patient with CRPS relapses with swelling of the wrist.
\end{abstract}

\section{Keywords}

Septic Arthritis, Complex Regional Pain Syndrome (CRPS), Hand

\section{Introduction}

Septic arthritis of the joint of the hand and wrist is unusual. The most common causative event is penetrating trauma. Other causes include immunocompromised patients, animal bites, or contiguous spread from an adjacent infectious site. Because of the small number of reports and limited clinical evidence, septic arthritis of the hand is difficult to diagnose in the initial phase, and acute septic arthritis may be misdiagnosed as cellulitis or flexor tenosynovitis. This delay in diagnosis may allow joint destruction to occur, and functional impairment may then remain after infection is resolved. Therefore, early diagnosis and treatment are particularly important.

Complex regional pain syndrome (CRPS) is a condition in which sharp pain is prolonged after injuries such as bone fracture and nerve damage. There are case reports of tuberculous arthritis of the hand during treatment of CRPS; however, the causal relationship of wrist joint inflammation with CRPS is unclear. Careful 
follow-up is necessary for wrist joint inflammation if a patient with CRPS relapses with swelling of the wrist. Here, we report a case in which CRPS type I developed after right carpal bone fracture and complicated with septic arthritis of the hand. We also provide a literature review. Informed consent for presentation of this case was obtained from the patient.

\section{Case Report}

The patient was an 86-year-old male with a chief complaint of pain and swelling in the right wrist. His medical history included pulmonary tuberculosis. He was diagnosed with fracture in the right trapezoid and capitate bones due to a fall and underwent conservative therapy with casts. However, he had significant pain in the right arm and fingers one month after injury and was diagnosed with CRPS because bone atrophy was found in the hand and around the wrist on plain radiographs. Conservative thermotherapy (oral administration of extracts from rabbit skin inflamed by the vaccinia virus) for CRPS was performed and swelling was gradually relieved.

Swelling in the right wrist recurred 7 months after injury. No marked contusion or sting was found, but bone destruction in the distal radius and carpal bone was apparent on plain radiographs (Figure 1). The white blood cell (WBC) count was $6600 / \mu \mathrm{L}$ and the C-reactive protein (CRP) level was $1.04 \mathrm{mg} / \mathrm{dL}$ in blood tests, showing a slight inflammatory response. Group $\mathrm{G}$ hemolytic streptococci were detected by arthrocentesis and the patient was diagnosed with septic arthritis of the hand. Joint culture, an interferon-gamma (IFN- $\gamma$ ) release assay, and a polymerase chain reaction (PCR) assay were conducted, but $\mathrm{Myco}$ bacterium tuberculosis was not detected, which excluded tuberculous arthritis of the hand.

The patient was diagnosed with septic arthritis of the hand and given cefcapene pivoxil hydrochloride at an oral dose of $300 \mathrm{mg} /$ day for one week, and minomycin, to which the bacteria were highly sensitive based on a culture result, at an oral dose of $200 \mathrm{mg} /$ day for 3.5 weeks. Antibiotics were withdrawn because the patient developed diarrhea, which was probably an adverse reaction to the antibiotics. Pain, swelling and redness in the right wrist were relieved, WBC was

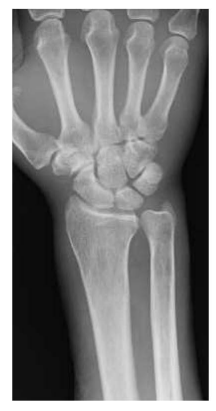

At time of injury

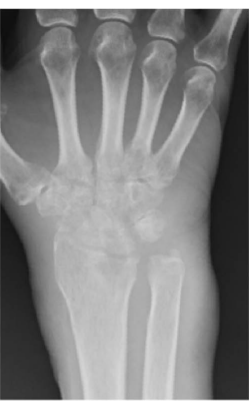

At diagnosis CRPS

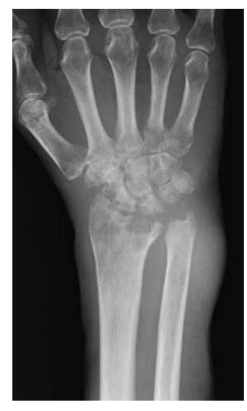

At diagnosis of septic arthritis

Figure 1. Plain radiograph changes. 
$4700 / \mu \mathrm{L}$ and CRP was $0.01 \mathrm{mg} / \mathrm{dL}$, showing improvement of the inflammatory response (Figure 2). Plain radiographs showed no progression of bone destruction, and osteosclerosis and infection were resolved. At final follow-up (13 months), a limited range of motion (ROM) (wrist: dorsal flexion $45^{\circ}$, volar flexion $30^{\circ}$, pronation $70^{\circ}$, supination $90^{\circ}$ ) remained, but the patient had no difficulty with daily activities (Figure 3 ).

\section{Discussion}

There are few case reports of purulent joint inflammation in the wrist [1] [2] [3]. This disease shows no gender difference in incidence, and age at onset ranges from children to the elderly. Of 141 cases of purulent joint inflammation reported by Kelly et al. [1], only 5 occurred in the wrist. The disease etiology is classified into extrinsic causes, such as trauma, and intrinsic causes, such as diabetes, malignant tumor and immunodeficiency. The major pathogenic bacteria are Staphylococcus aureus, and minor causative bacteria include group G hemolytic streptococcus, $P$. aeruginosa and Mycobacterium tuberculosis.

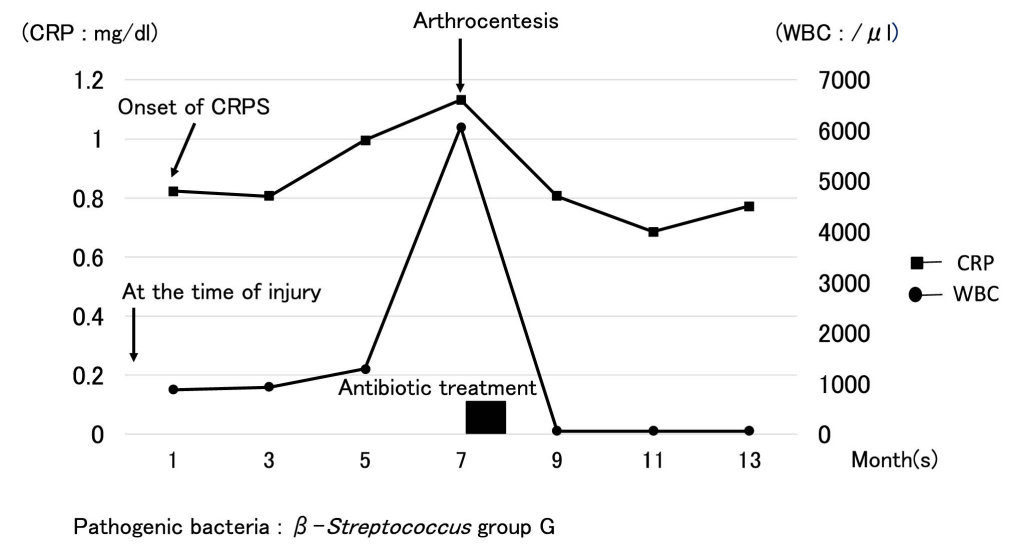

Figure 2. Disease course.
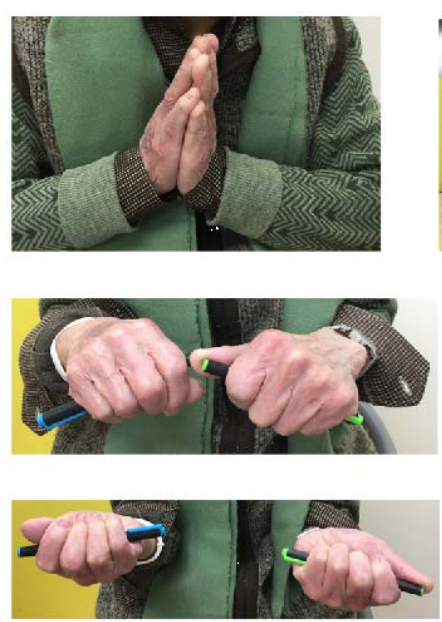
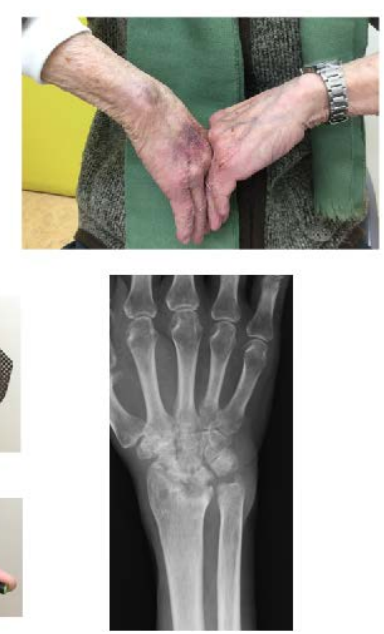

Figure 3. Photographs showing range of motion. Plain radiograph at the final examination (after 13 months). 
Local findings of pain, swelling, redness and limited ROM of the wrist, and an increased inflammatory response and elevated WBC count in blood are useful for diagnosis. A nitroblue tetrazolium (NBT) reduction test can also be used to diagnose infections based on an increase in NBT-positive cells of less than 10\% in healthy individuals with neutrophils affected by infection. However, this test may give false-negative and false-positive results. Elderly patients are sometimes diagnosed with hand osteoarthritis and rheumatoid arthritis [4] [5] and there are many cases in which diagnosis of septic arthritis of the hand is difficult.

Rashkoff et al. [2] reported 23 patients with septic arthritis of the hand and pointed out the importance of early surgical drainage, antibiotic administration, and training of ROM. Ten patients with excellent or good outcomes underwent surgical drainage within $10 \mathrm{~h}$ after onset of symptoms, whereas 23 patients with fair or poor outcomes were surgically drained more than $13 \mathrm{~h}$ after onset. Murray et al. [6] proposed the following treatment principles for septic arthritis of the hand: early diagnosis, surgical drainage, effective antibiotic administration, and early training of joint ROM. Terabe et al. [7] showed that patients with staphylococcal septic arthritis caused by $P$. aeruginosa who did not undergo surgical drainage, but received 4-week antibiotic treatment and 8-week external fixation, still had limited volar flexion and inferior wrist function, despite resolution of infection. Ochi et al. [3] reported a case of tuberculous arthritis of the hand that occurred during treatment of CRPS, in which limited ROM remained despite improvement of pain and infection by surgical treatment after difficulty with diagnosis.

To our knowledge, the current report is the first to describe CRPS complicated with septic arthritis of the hand. Our patient had allodynia-like pain, a burning sensation, marked edema, changed skin color, body hair on the wrist, and decalcified bone images on plain radiographs. He was diagnosed with CRPS using the CRPS score of Gibbons et al. [8] and treated conservatively with thermotherapy, using oral extracts from rabbit skin inflamed by the vaccinia virus. This led to gradual relief of swelling. During treatment, diffuse swelling relapsed in the peripheral forearm and inflammatory markers slightly increased in blood tests. Since bone destruction and bone atrophy were also shown by plain radiography, arthrocentesis was conducted to examine infection; consequently, hemolytic streptococci were detected in cultures and the patient was diagnosed with septic arthritis of the hand. He had no marked antecedent trauma. Group G hemolytic streptococci, which are relatively rare bacteria, were detected as pathogenic bacteria. Based on a previous case report of tuberculous arthritis of the hand [3], the slight inflammatory findings in our patient, and his history of pulmonary tuberculosis, we examined the presence of $M$. tuberculosis and excluded this species as pathogenic bacteria.

The patient underwent conservative therapy with rest and antibiotics for about 4 weeks. Surgery was not performed because of age, a contraindication for CRPS, and marked bone destruction in plain radiographs. Pain and swelling were relieved and infection was resolved. Limited ROM remained, but the pa- 
tient had no difficulty with daily activities. The causal relationship between CRPS and arthritis of the hand is unclear, but patients with CRPS sometimes develop arthritis of the hand, including tuberculous arthritis, during treatment. Therefore, careful follow-up of a suspected complication of infection during treatment of CRPS is necessary in a case with bone destruction and bone atrophy on plain radiographs.

\section{Conclusion}

We treated a patient with an extremely rare case of septic arthritis of the hand that developed during treatment of CRPS. The causal relationship between CRPS and onset of arthritis of the hand is unclear; however, careful follow-up is needed for a suspected complication of infection in a case of CRPS with relapsed swelling and bone destruction and atrophy in plain radiographs.

\section{Conflicts of Interest}

The authors declare no conflicts of interest regarding the publication of this paper.

\section{References}

[1] Kelly, P.J. (1975) Bacterial Arthritis in the Adult. Orthopedic Clinics of North America, 6, 973-981.

[2] Rashkoff, E.S., et al. (1983) Septic Arthritis of the Wrist. JBJS, 65-A, 824. https://doi.org/10.2106/00004623-198365060-00015

[3] Ochi, K., Horiuchi, Y., kawashima, H., et al. (2010) Tuberculosis of a Wrist Joint Which Was Similar to Complex Regional Pain Syndrome, a Case Report. Orthopedics, 61, 439-442.

[4] Kapten, I. (1973) Septic Arthritis Complicating Rheumatoid Arthritis. Annals of Internal Medicine, 54, 461-472.

[5] Russell, A.S. and Ansell, B.M. (1972) Septic Arthritis. Annals of the Rheumatic Diseases, 31, 40-44. https://doi.org/10.1136/ard.31.1.40

[6] Murray, P.M. (1998) Septic Arthritis of the Hand and Wrist. Hand Clinics, 14, 579-587.

[7] Kenya. T., et al. (2007) Septic Arthritis of the Wrist due to Pseudomonas Aeruginosa. Journal of Orthopaedic Trauma, 50, 489-490.

[8] Gibbons, J.J. and Wilson, P.R. (1992) RSD Score: Criteria for the Diagnosis of Reflex Sympathetic Dystrophy and Causalgia. The Clinical Journal of Pain, 8, 260-263. 